\title{
Níveis de sombreamento e tipos de malha no crescimento e produção de óleo essencial de hortelã-pimenta
}

\author{
Andressa G Costa ${ }^{1}$; Jorge H Chagas²; Suzan KV Bertolucci ${ }^{3}$; José EBP Pinto ${ }^{3}$

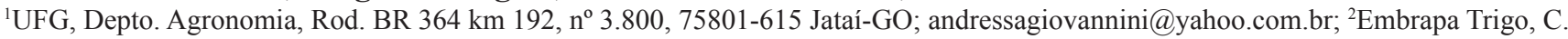 \\ Postal 451, 99001-970 Passo Fundo-RS; jorge.chagas@cpac.embrapa.br; ${ }^{3}$ UFLA, Depto. Agricultura, C. Postal 3037 37200-000 Lavras- \\ MG; suzan@dag.ufla.br; jeduardo@dag.ufla.br (autor para correspondência)
}

\section{RESUMO}

Objetivou-se avaliar o crescimento vegetativo da planta, o teor, o rendimento e a composição química do óleo essencial de hortelã- pimenta (Mentha piperita), cultivada sob diferentes malhas e níveis de irradiância. As plantas foram cultivadas sob a malha preta e aluminizada com diferentes níveis de sombreamento $(0,30,50$ e $70 \%)$. Realizou-se a colheita quatro meses após o plantio. A área foliar (AF), razão peso foliar (RPF) e o teor do óleo essencial não diferiram significativamente. A biomassa seca da folha (BSF), do caule (BSC), da raiz (BSR), total (BST), razão da área foliar (RAF) e área foliar específica (AFE) das plantas cultivadas em cada malha apresentaram comportamentos distintos nos diferentes níveis de sombreamento. O crescimento das plantas de hortelã-pimenta foi $12,5 \%$ menor na malha preta $\left(32,6\right.$ g planta $\left.^{-1}\right)$ e quanto maior o sombreamento menor foi a produção de biomassa nas malhas aluminizada e preta $(34,2$ e 17,7 g planta $^{-1}$, respectivamente). O rendimento de óleo essencial foi de 1,26 mL planta ${ }^{-1}$ para malha preta e $1,34 \mathrm{~mL}_{\text {planta }}{ }^{-1}$ para malha aluminizada. $\mathrm{O}$ crescimento e rendimento de óleo essencial foram superiores no cultivo a pleno sol do que nas malhas e seus diferentes níveis de sombreamento. A hortelã-pimenta respondeu distintamente aos níveis de sombreamento e malhas quanto aos teores dos monoterpenos majoritários; assim, sugere-se o cultivo a pleno sol.

Palavras-chave: Mentha piperita, composição química, biomassa, tela aluminizada.

\section{ABSTRACT}

Shading levels and net type on vegatative growth and essential oil production of peppermint

The objective was to evaluate plant growth, content, yield and chemical composition of essential oil of peppermint (Mentha piperita) grown under different nets and intensities of light. The plants were grown under the black net and aluminized net with different shading levels $(0,30,50$ and $70 \%)$. The harvest was done 4 months after plating. Foliar area, foliar weight ratio and essential oil content were not statistically different. The dry biomass of leaves, shoots, roots, total, ratio of foliar area and specific foliar area of plants cultivated under each net showed distinct responses in different shading levels. The peppermint plant growth was $12.5 \%$ lower in black net $(32.6$ g plant $\left.^{-1}\right)$; more shading resulted in less biomass production in the aluminized and black nets (34.2 and 17.7 g plant $^{-1}$, respectively). The essential oil yield was $1.26 \mathrm{~mL}$ plant $^{-1}$ for black net and 1.34 $\mathrm{mL}$ plant $^{-1}$ for aluminized net. The growth and essential oil yield were higher under full sunlight than under nets of distinct shading levels. The peppermint replied distinctly to nets and their shading levels for the production of majority monoterpenes thus suggesting its growth in full sunlight.

Keywords: Mentha piperita, chemical composition, biomass, aluminized net.

(Recebido para publicação em 26 de julho de 2013; aceito em 24 de abril de 2014) (Received on July 26, 2013; accepted on April 24, 2014)

\begin{abstract}
A s mentas são cultivadas como ervas, cujas folhas podem ser secas e usadas como flavorizantes; seu óleo essencial é usado como aromatizante pelas indústrias farmacêuticas em fragrâncias, na medicina e como condimento alimentar (Lorenzo et al., 2002). O mentol e a mentona são os principais componentes do óleo essencial de Mentha spp. e os de maior valor econômico, embora sejam conhecidos mais de 200 componentes presentes nos óleos do gênero Mentha (Pegoraro et al., 2010). A biossíntese de monoterpenos é regulada pela interação de fatores ontogenéticos e ambientais, incluindo disponibilidade de nutrientes, temperatura, fotoperíodo e radiação (Behn et al., 2010). A luz é o principal fator que controla o crescimento, o desenvolvimento e o metabolismo das
\end{abstract}

plantas, sendo afetadas pela irradiância em todos os estágios do seu crescimento (Chang et al., 2008; Costa et al., 2012).

As malhas termorrefletoras por serem revestidas de alumínio e terem fios retorcidos, fazem com que a temperatura do ambiente abaixe de 10 a $20 \%$, fornecendo, em média, 15\% de luz difusa ao ambiente, não afetando os processos fotossintéticos e, ainda, promovendo o sombreamento (Vischi Filho, 2002). A diminuição da temperatura e a adição de luz difusa propiciam microclima responsável pelo incremento da matéria seca, traduzida pela precocidade em experimentação com mudas de citrus (Polysack..., 2008). Por outro lado, o cultivo protegido tradicional, além de exigir estrutura onerosa, proporciona efeito estufa pela cobertura plástica, que é prejudicial em épocas quentes do ano. As telas de sombreamento, na maioria das vezes, não proporcionam níveis adequados de luz, afetando o desenvolvimento e a produção (Corrêa, 2008).

O objetivo deste trabalho foi avaliar a influência da malha preta e aluminizada com diferentes níveis de sombreamento no crescimento, teor e produção de óleo essencial de hortelã-pimenta (Mentha piperita).

\section{MATERIAL E MÉTODOS}

O experimento foi desenvolvido no município de Lavras-MG (21 ${ }^{\circ} 14^{\prime} 07^{\prime}$ S; 4458'22'O; $879 \mathrm{~m}$ de altitude). De acordo com a classificação climática de Köppen, o clima regional é do tipo Cwa, 
mas apresenta características de Cwb com duas estações bem definidas, uma fria e seca, de abril a setembro, e outra quente e úmida, de outubro a março.

O experimento foi conduzido de março a julho de 2009. As condições climáticas da área experimental apresentaram temperaturas médias mensais de $20,9^{\circ} \mathrm{C}$, observando-se mínimas de 13,0 e máxima de $31,6^{\circ} \mathrm{C}$, e precipitação média mensal de 136,26 mm durante o experimento. A umidade relativa do ar média foi de $74 \%$, variando entre 66 e $80 \%$, e a insolação média foi de 6,3 horas, variando entre 4,0 e 7,5 h. Estes dados foram coletados na Estação Climatológica Principal de Lavras, localizada a, aproximadamente, um quilometro da área experimental.

As mudas foram obtidas a partir de mini estacas apicais (aproximadamente $5 \mathrm{~cm}$ de comprimento) de plantas matrizes do Horto de Plantas Medicinais da Universidade Federal de Lavras e enraizadas em bandejas de polipropileno expandido de 128 células utilizando-se substrato comercial. Após o enraizamento as mudas foram transplantadas para vasos de plásticos de $10 \mathrm{~L}$ de capacidade, contendo uma mistura de terra, areia e esterco bovino (3:1:1). A mistura foi submetida à análise química em laboratório da UFLA.

A caracterização química da mistura foi obtida conforme Embrapa (1997). As características químicas da mistura de terra, areia e esterco $(3: 1: 1)$ foram: $\mathrm{pH}$ em água $=6,5 ; \mathrm{P}$ e $\mathrm{K}\left(\mathrm{mg} \mathrm{dm}^{-3}\right)=$ 55,5 e 284,0, respectivamente; $\mathrm{Ca}^{2+}$, $\mathrm{Mg}^{2+}, \mathrm{Al}^{3+}, \mathrm{H}+\mathrm{Al}\left(\mathrm{cmol}_{\mathrm{c}} \mathrm{dm}^{-3}\right)=3,4 ; 1,4$; 0,$0 ; 1,3$, respectivamente; saturação de bases $\mathrm{V}(\%)=81,0$; matéria orgânica $\left(\right.$ dag $\left.\mathrm{kg}^{-1}\right)=1,3$.

$\mathrm{O}$ experimento foi realizado em delineamento inteiramente casualizado, com esquema fatorial, $2 \times 4$, sendo duas malhas de cobertura (preta e termorrefletora aluminizada), e quatro níveis de sombreamento $(0,30,50$ e $70 \%)$, com cinco repetições, onde cada parcela foi constituída de quatro plantas.

A colheita foi realizada após 120 dias de cultivo, quando cada planta foi separada em raiz, caule e folha para secagem em estufa com ventilação forçada a $40^{\circ} \mathrm{C}$ até peso constante. A massa do material vegetal seco foi obtida para de- terminação da biomassa seca de folhas (BSF, g/planta), caule (BSC, g/planta), raízes (BSR, g/planta) e total da planta (BST, g/planta), determinando-se também a relação raiz:parte aérea ( $\mathrm{R} / \mathrm{PA})$. Após a colheita, avaliou-se a área foliar (AF, $\mathrm{cm}^{2} /$ planta), medida com auxílio de Medidor Eletrônico de área foliar, modelo LI-3100-LICOR. A partir da qual se obteve os parâmetros fisiológicos de razão de área foliar ( $\left.\mathrm{RAF}, \mathrm{cm}^{2} / \mathrm{g}\right)$, área foliar específica (AFE, $\mathrm{cm}^{2} / \mathrm{g}$ ), razão do peso foliar (RPF), peso específico foliar (PEF, $\mathrm{mg} / \mathrm{cm}^{2} /$ planta) de acordo com Benincasa (2003).

O óleo essencial foi extraído de $20 \mathrm{~g}$ de folhas secas da hortelã-pimenta pelo processo de hidrodestilação em aparelho de Clevenger modificado, durante 120 minutos. O óleo foi separado por decantação por $5 \mathrm{~min}$, sendo recolhido o sobrenadante. Foram determinados o teor $(\mu \mathrm{L} / 100 \mathrm{~g} \mathrm{BSF})$, o rendimento (mL/ planta) e a composição química do óleo. As análises da composição química foram realizadas por meio de uma amostra composta de alíquotas equivolumétricas do óleo volátil das repetições de cada tratamento.

As análises quantitativas do óleo foram realizadas por cromatografia em fase gasosa acoplado a um detector de ionização de chama de hidrogênio (CG-DIC) em um sistema Agilent ${ }^{\circledR} 7890 \mathrm{~A}$ equipado com coluna capilar de sílica fundida HP-5 (30 m de comprimento x 0,25 mm de diâmetro interno x 0,25 $\mu \mathrm{m}$ de espessura do filme). O gás Hélio foi utilizado como gás de arraste com fluxo de 1,0 mL/min; as temperaturas do injetor e do detector foram mantidas em 220 e $240^{\circ} \mathrm{C}$, respectivamente. A temperatura inicial do forno foi de $60^{\circ} \mathrm{C}$, isotérmico por $1,5 \mathrm{~min}$, seguido por uma rampa de temperatura de $3^{\circ} \mathrm{C} /$ min até $240^{\circ} \mathrm{C}$, seguida de uma rampa de $10^{\circ} \mathrm{C} / \mathrm{min}$ até $270^{\circ} \mathrm{C}$. O óleo foi diluído em acetato de etila $(1 \%, \mathrm{v} / \mathrm{v})$ e injetado automaticamente no cromatógrafo empregando volume de injeção de $1,0 \mu \mathrm{L}$, no modo split a uma razão de injeção de 1:50. A análise quantitativa foi obtida pela integração do cromatograma total de íons (TIC) e o teor dos constituintes eluídos expressos como \% de área relativa das áreas dos picos. As análises qualitativas do óleo foram realizadas por cromatografia gasosa acoplada à espectrometria de massas (CG-EM), utilizando-se um equipamento Agilent ${ }^{\circledR}$ 5975C, operado por ionização de impacto eletrônico a $70 \mathrm{eV}$, em modo varredura, a uma velocidade de 1,0 scan/s, com um intervalo de aquisição de massas de 40-400 m/z. As condições cromatográficas foram as mesmas empregadas nas análises quantitativas.

Para análise estatística dos dados, foi utilizado o programa Sisvar ${ }^{\circledR}$ (Ferreira, 2007). As médias entre os tratamentos foram submetidas à análise de variância, pelo teste de $\mathrm{F}$ e aplicado o teste de Scott-Knott $(\mathrm{p}<0,05)$.

\section{RESULTADOS E DISCUSSÃO}

O uso das malhas preta e aluminizada com diferentes níveis de sombreamento influenciaram o crescimento das plantas de hortelã-pimenta. Houve interação significativa entre as malhas e os níveis de sombreamento para as variáveis de crescimento $\mathrm{BSF}, \mathrm{BSC}, \mathrm{BSR}$, BST, RAF e AFE e rendimento do óleo essencial. Para as variáveis AF, RPF, PEF e teor de óleo essencial não foram observadas diferenças significativas.

A BSF apresentou correlação linear com decréscimo de 0,2 g planta $^{-1}$ a cada $10 \%$ de aumento do sombreamento nas plantas cultivadas na malha. Sob a malha preta observou-se uma correlação quadrática, havendo produção máxima no nível de 13 a 14,2\% de sombreamento, decaindo a BSF com o aumento do sombreamento (Figura 1A). Resultados similares foram observados por Chagas et al. (2010b) em Mentha arvensis, que obtiveram maior produção de biomassa seca quando as plantas foram cultivadas a pleno sol, diminuindo linearmente com o aumento do sombreamento.

As plantas cultivadas nas malhas apresentaram resultados distintos para BSC. A malha aluminizada obteve maior acúmulo com $70 \%$ de sombreamento (18,38 g/planta) (Figura 1B). O maior acúmulo de BSC com a diminuição da radiação incidente é uma característica de plantas competitivas, o que pode demonstrar uma forma de escape ao déficit de luz, no entanto, isto não foi observado na malha preta, onde há um 


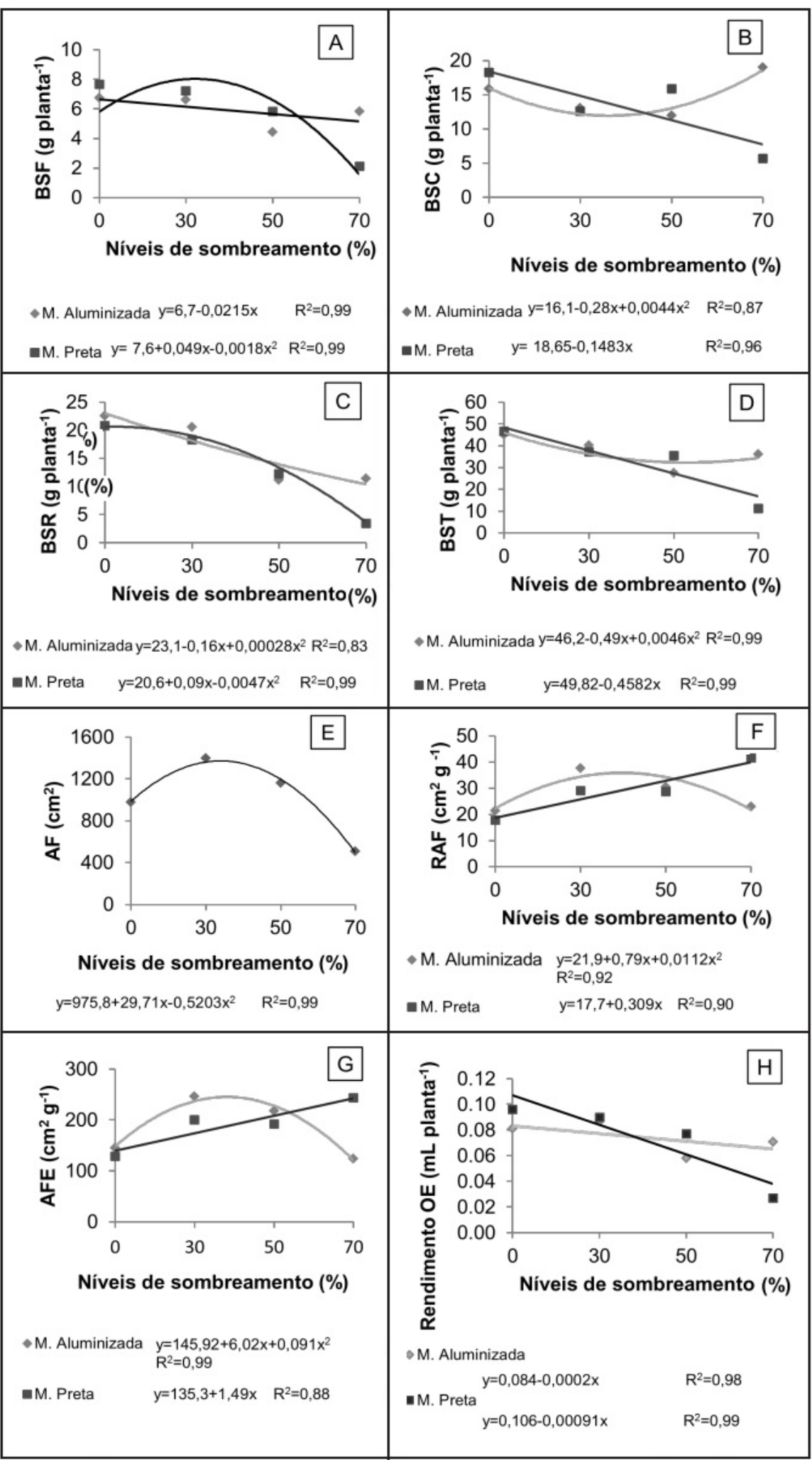

Figura 1. Desdobramento da interação das variáveis biomassa seca de folha (BSF, A), de caule (BSC, B), de raiz (BSR, C) e total (BST, D), área foliar (AF, E), razão da área foliar (RAF, F), área foliar específica (AFE, G) e rendimento do óleo essencial (H) de plantas de hortelã-pimenta cultivadas em diferentes níveis de sombreamento na malha aluminizada e na malha preta [interaction of parameters of leaf dry biomass (BSF, A), shoot (BSC, B), root $(\mathrm{BSR}, \mathrm{C})$ and total (BST, D), foliar area (AF, E), foliar area ratio (RAF, F), specific foliar area (AFE, $G$ ) and yield of essential oil $(\mathrm{H})$ of peppermint plants cultivated under differents shading levels of aluminized net and black net]. Lavras, UFLA, 2009. decréscimo na BSC com o aumento do sombreamento.

A BSR nas plantas cultivadas na malha aluminizada e preta manteve correlação quadrática, resultando em máxima produtividade de raiz quando cultivadas a plenos sol (23,1 e 20,6 g/planta, respectivamente) decrescendo com o aumento do sombreamento (Figura 1C). Segundo Castro et al. (1998), existe uma alocação preferencial de fotoassimilados para o sistema radicular nas plantas cultivadas a pleno sol em detrimento de plantas cultivadas sob baixas condições de luminosidade, o que corrobora com os resultados observados, tanto na malha preta como na aluminizada. Almeida \& Mundstock (2001) explicam essa queda na alocação de fotoassimilados para as raízes com a diminuição da luminosidade, como sendo uma resposta a atributos que melhoram o ganho de carbono sobre irradiância reduzida.

ABST decresceu com o aumento em ambas as malhas, atingindo máxima produção quando não houve sombreamento (46,0 g/planta) (Figura 1D). Resultados similares foram observados por Chang et al. (2008) em Ocimum basilicum e por Chagas et al. (2010b) em M. arvensis, havendo maior produção de biomassa seca quando cultivadas a pleno sol, demonstrando que estas espécies apresentam melhor crescimento em ambientes ensolarados, não sendo adaptadas para cultivo em consórcio com espécies de porte mais alto, devido ao sombreamento proporcionado por estas.

Comparando as variáveis de crescimento entre as malhas preta e aluminizada (Tabela 1), nota-se que houve um ganho de BSC, BSR e BST no cultivo sob malha aluminizada em detrimento à malha preta. Segundo Vischi Filho (2002), o uso da malha termorrefletora revestida de alumínio reduz a temperatura ambiente e fornece, em média, $15 \%$ de luz difusa ao ambiente, o que pode contribuir para maior acúmulo de biomassa seca, como foi observado neste experimento.

Visando maximização da absorção luminosa, plantas sombreadas podem ter área superficial de 20 a $80 \%$ maior do que folhas crescidas ao sol, decorrente da ampliação da superfície fotossinte- 
Tabela 1. Biomassa seca de folhas (BSF), caule (BSC), raiz (BSR), total (BST), relação raiz/parte aérea (R/PA), área foliar (AF), razão do peso foliar (RPF), razão da área foliar (RAF), área foliar específica (AFE), peso específico foliar (PEF), rendimento e teor de óleo essencial (OE) de hortelã-pimenta cultivada sob níveis de sombreamento e tipos de malha [dry biomass of leaves (BSF), shoot (BSC), root (BSR), total (BST), ratio of root/shoot (R/ PA), foliar area (AF), foliar area ratio (RPF), specific foliar area (RAF), foliar specific mass (AFE), yield and content of essential oil of peppermint cultivated under shading levels and net types]. Lavras, UFLA, 2009.

\begin{tabular}{|c|c|c|c|c|}
\hline \multirow{2}{*}{ Variável } & \multicolumn{2}{|c|}{ Malha } & \multirow{2}{*}{ Média } & \multirow{2}{*}{ CV $(\%)$} \\
\hline & aluminizada & preta & & \\
\hline BSF (g/planta) & $5,71 \mathrm{~A}$ & $5,91 \mathrm{~A}$ & 5,81 & 15,76 \\
\hline BSC (g/planta) & $14,96 \mathrm{~A}$ & $13,09 \mathrm{~B}$ & 14,03 & 12,98 \\
\hline BSR (g/planta) & $16,42 \mathrm{~A}$ & $14,20 \mathrm{~B}$ & 15,31 & 15,14 \\
\hline BST (g/planta) & $37,29 \mathrm{~A}$ & $32,65 \mathrm{~B}$ & 34,96 & 9,13 \\
\hline $\mathrm{R} / \mathrm{PA}$ & $0,81 \mathrm{~A}$ & $0,72 \mathrm{~A}$ & 0,76 & 19,41 \\
\hline $\mathrm{AF}\left(\mathrm{cm}^{2} /\right.$ planta $)$ & $1079,67 \mathrm{~A}$ & 940,88 B & 1010,27 & 16,05 \\
\hline RPF (g/g) & $0,16 \mathrm{~A}$ & $0,17 \mathrm{~A}$ & 0,16 & 15,51 \\
\hline $\operatorname{RAF}\left(\mathrm{cm}^{2} / \mathrm{g}\right)$ & $28,16 \mathrm{~A}$ & $29,29 \mathrm{~A}$ & 28,72 & 28,49 \\
\hline $\operatorname{AFE}\left(\mathrm{cm}^{2} / \mathrm{g}\right)$ & $183,51 \mathrm{~A}$ & $191,06 \mathrm{~A}$ & 187,28 & 21,80 \\
\hline PEF (mg/cm/planta) & $6,15 \mathrm{~A}$ & $5,93 \mathrm{~A}$ & 6,04 & 19,56 \\
\hline Rendimento OE (mL/planta) & $0,07 \mathrm{~A}$ & $0,07 \mathrm{~A}$ & 0,07 & 25,95 \\
\hline Teor OE $(\mu \mathrm{L} / 100 \mathrm{~g}$ BSF $)$ & $1,26 \mathrm{~A}$ & $1,34 \mathrm{~A}$ & 1,30 & 24,18 \\
\hline
\end{tabular}

Médias seguidas por letras distintas nas linhas diferem entre si ao nível de 5\% de probabilidade pelo teste de Scott-Knott (means followed by different letters in rows differ at $5 \%$ probability by Scott-Knott test).

tizante na planta (Nobel, 1991; Scalon et al., 2001). A AF apresentou resposta quadrática nos diferentes níveis de sombreamento ocorrendo uma redução a partir de 28\% de sombreamento (Figura 1E). Chagas et al. (2010a) observaram também que, mesmo as plantas de Mentha arvensis acumulando menos biomassa seca nas folhas com o aumento do sombreamento, elas conseguiram expandir sua área foliar total até um sombreamento de $28 \%$ sob malha preta e $30,7 \%$ sob a malha aluminizada, resultado este similar ao encontrado neste experimento, podendo-se observar área máxima $\left(1399,92 \mathrm{~cm}^{2}\right)$ quando as plantas foram cultivadas com $28,6 \%$ de sombreamento (Figura 1E). A redução no nível de radiação fez com que as plantas aumentassem a proporção investida no crescimento em área foliar. Este aumento pode decorrer do aumento na expansão celular ao longo da lâmina foliar.

A RAF das plantas, quando cultivadas na malha aluminizada, apresentou correlação quadrática, havendo uma redução com o aumento do sombreamento (Figura 1F). A diminuição da demais partes das plantas aumentaram com a diminuição da radiação solar. Para as plantas de hortelã-pimenta esta proporção não foi alterada pelo uso das malhas nem pelos níveis de sombreamento, visto que não foram observadas diferenças significativas.

AAFE é um indicativo da espessura da folha e estima a proporção relativa da superfície assimilatória e dos tecidos de sustentação e condutores da folha (Benincasa, 2003). A AFE das plantas de hortelã-pimenta apresentou resposta quadrática quando cultivadas na malha aluminizada e linear quando cultivadas sob malha preta (Figura 1G). O que pode demonstrar que a maior radiação incidente proporciona maior espessura das folhas em hortelã-pimenta. Resultado observado também em Mentha suaveolens (Amaral, 2007), em Ocimum basilicum (Chang et al., 2008) e em Mentha arvensis (Chagas et al., 2010a), que indicaram uma redução na espessura das folhas com o aumento do sombreamento. Isto pode ser caracterizado como uma adaptação das plantas tolerantes ao sol, sendo que a redução pode estar associada a um menor volume de células.

O teor do óleo essencial não foi afetado pelos níveis de sombreamento e malhas; os valores obtidos indicaram concentrações entre 1,2 a $1,3 \mu \mathrm{L} 100 \mathrm{~g}^{-1}$ BSF (Tabela 1). O rendimento, como é dependente do acúmulo de biomassa nas folhas, apresentou resultado similar àquele observado para BSF. Houve um decréscimo linear no rendimento do óleo essencial em ambas malhas à medida que os níveis de sombreamento aumentaram, sendo na malha preta a redução do rendimento mais acentuada em comparação com a malha aluminizada. Consequentemente, observou-se que o máximo rendimento para ambas as malhas foi no tratamento a pleno sol (Figura 1H).

Em Ocimum basilicum o aumento da radiação incidente não aumentou o rendimento de óleo, mas afetou a percentagem relativa dos componentes do óleo, ocasionando um aumento no linalol e eugenol, enquanto que o metileugenol aumentou com um maior sombreamento (Chang et al., 2008). Amaral (2007) não observou a influência da intensidade luminosa nos teores de óleo essencial 
Tabela 2. Análise química do óleo essencial de hortelã-pimenta sob níveis de irradiação e tipos de malha (chemical analysis of essential oil of peppermint under irradition levels and net types). Lavras, UFLA, 2009.

\begin{tabular}{|c|c|c|c|c|c|c|c|c|}
\hline \multirow{2}{*}{ Composto } & \multirow{2}{*}{ IK } & \multirow{2}{*}{ Pleno sol } & \multicolumn{3}{|c|}{ Malha aluminizada (\%) } & \multicolumn{3}{|c|}{ Malha preta (\%) } \\
\hline & & & 30 & 50 & 70 & 30 & 50 & 70 \\
\hline limoneno & 1012 & 2,63 & 2,14 & 2,13 & 2,67 & 2,47 & 2,64 & 2,31 \\
\hline 1,8-cineol & 1015 & 0,86 & 0,82 & 0,32 & 1,05 & 0,96 & 0,87 & 0,84 \\
\hline g-terpineno & 1050 & 0,63 & 0,85 & 0,46 & 0,89 & 0,93 & 0,93 & 0,81 \\
\hline mentona & 1142 & 8,55 & 7,39 & 3,46 & 3,01 & 6,77 & 5,26 & 12,59 \\
\hline mentofurano & 1153 & 21,13 & 28,02 & 28,75 & 35,72 & 30,34 & 34,37 & 24,37 \\
\hline mentol & 1164 & 25,01 & 25,69 & 13,24 & 15,21 & 25,52 & 15,23 & 26,82 \\
\hline pulegona & 1237 & 1,02 & 1,23 & 1,12 & 1,15 & 1,25 & 1,96 & 3,20 \\
\hline acetato de neomentila & 1278 & 2,38 & 1,56 & 3,74 & 3,14 & 1,72 & 2,10 & 1,59 \\
\hline acetato de mentila & 1299 & 32,20 & 26,87 & 41,11 & 34,02 & 24,53 & 27,78 & 21,03 \\
\hline Total & & 94,43 & 94,58 & 94,36 & 96,86 & 94,50 & 91,14 & 93,57 \\
\hline
\end{tabular}

IK: índice de Kovats calculado em relação à retenção de $n$-alcanos $\left(\mathrm{C}_{8}\right.$ a $\left.\mathrm{C}_{20}\right)$ em coluna HP-5 (IK: Kovats indice calculated in relation to the retention of n-alkanes $\left(\mathrm{C}_{8}\right.$ to $\left.\mathrm{C}_{20}\right)$ on HP-5 column).

de Mentha suaveolens. Contudo nestes trabalhos, devido à maior produção de biomassa seca das folhas nas plantas sombreadas $(50 \%)$, o rendimento do óleo essencial foi maior nas condições de sombreamento.

As análises químicas das amostras de óleo essencial de hortelã pimenta identificaram, em média, cerca de $94,2 \%$ dos constituintes presentes no óleo, o qual é formado totalmente por monoterpenos (Tabela 2). Os constituintes majoritários são representados pela mentona, mentofurano, mentol e acetato de neomentila, os quais constituem, em média, cerca de $86 \%$ do conteúdo total de constituintes identificados no óleo. Os óleos essenciais limoneno, 1,8-cineol, g-terpineno, pulegona e acetato de neomentila são os constituintes minoritários identificados no óleo de hortelã-pimenta em concentrações entre 0,32 a $3,74 \%$.

Aconcentração de mentona (12,59\%) e mentol $(26,82 \%)$ aparentemente foram maiores quando a hortelã-pimenta foi cultivada sob malha preta com $70 \%$ de sombreamento. Porém, os teores de mentol não variaram consideravelmente entre os cultivos a pleno sol, nas malhas termorrefletora $30 \%$ e malha preta $30 \%$ $(25,01 ; 25,69$ e 25,52 , respectivamente) em comparação à malha preta $70 \%$. Nos demais ambientes os teores de mentol variaram entre 13,24 a $15,23 \%$. O menor teor de mentofurano foi observado a pleno sol $(21,13 \%)$ e os maiores sob as malhas aluminizada $70 \%$, preta $30 \%$ e preta $50 \%(35,72 ; 30,34$ e 34,37 , respectivamente) (Tabela 2). Os efeitos das variações de luz e de temperatura podem influenciar no metabolismo primário e secundário das plantas, atuando na formação dos monoterpenos (Dolzhenko et al., 2010).

$\mathrm{Na}$ síntese do óleo essencial de hortelã-pimenta, a (+)-pulegona é precursora do mentol. Dependendo das condições ambientais, a (+)-pulegona pode reduzir a (-)-mentona e sintetizar mentol, através da pulegona redutase, ou oxidar a (+)-mentofurano, pela mentofurano sintase (Mahmoud \& Croteau, 2003). O acetato de mentila é o produto da acetilação do mentol (Maffei et al., 1999). Com base nisto, notou-se que no presente estudo as reações de oxidação-redução e acetilação da rota biossintética dos monoterpenos de hortelã-pimenta foram bastante influenciadas pelo nível de sombreamento e tipo de malha empregada no cultivo.

As plantas cultivadas sob a malha aluminizada com $50 \%$ de sombreamento favoreceram a conversão do mentol em acetato de mentila, pois apresentaram baixo teor de mentol $(13,24 \%)$ e elevados teores de acetato de mentila $(41,11 \%)$ (Tabela 2). Na malha aluminizada com $70 \%$ de sombreamento esse mesmo fenômeno parece ter ocorrido, porém houve um balanço entre a acetilação do mentol $(15,21 \%)$ a acetato de mentila $(34,02 \%)$ e a oxidação da pulegona para síntese do mentofurano $(35,72 \%)$.

Vale ressaltar ainda, a composição distinta do óleo das plantas cultivadas sob malha preta com $70 \%$ de sombreamento (Tabela 2). Nesse ambiente, nota-se um teor mais equilibrado dos quatro monoterpenos majoritários, conferindo uma diferenciação ao óleo em relação aos demais, por conter maiores concentrações de mentona $(12,59 \%)$ e mentol $(26,82 \%)$ e concentrações reduzidas de mentofurano $(24,37 \%)$ e acetato de mentila $(21,03 \%)$.

Burbott \& Loomis (1967) demonstraram que noites curtas e frias combinadas com altas intensidades de luz durante o dia aumentam a formação de mentona e diminuem o acúmulo de mentofurano e pulegona. A hortelã-pimenta mostra drásticas alterações no metabolismo em resposta a condições ambientais, incluindo a qualidade e quantidade de luz (Maffei et al., 1999; Behn et al., 2010; Dolzhenko et al., 2010). De acordo com Sacco (1987), as plantas de hortelã-pimenta são sensíveis ao fotoperíodo, ou seja, são plantas longodiurnas. A maturação e a qualidade do óleo poderiam representar um problema em áreas de menor latitude, resultando óleos essenciais com conteúdos elevados de mentofurano, componente indesejável no óleo essencial. Com isso, parece que o fotoperíodo associado com as variações ambientais influencia consideravelmente a composição de 
terpenos dessa espécie.

Pode-se concluir que o sombreamento promovido pelas malhas diminuiu o crescimento e o rendimento do óleo essencial, mas indicou que plantas de hortelã-pimenta respondem distintamente aos níveis de sombreamento e malhas quanto aos teores dos monoterpenos majoritários; assim, sugere-se o cultivo a pleno sol.

\section{AGRADECIMENTOS}

À Coordenação de Aperfeiçoamento de Nível Superior (CAPES), pela concessão da bolsa de estudos, ao Conselho Nacional de Desenvolvimento Científico (CNPq) e à Fundação de Amparo à Pesquisa do Estado de Minas Gerais (FAPEMIG), pela bolsa de produtividade, apoio técnico e pelo apoio financeiro.

\section{REFERENCIAS}

ALMEIDA ML; MUNDSTOCK CM. 2001. O afilhamento da aveia afetado pela qualidade de luz em plantas sob competição. Ciência Rural 31: 393-400.

AMARAL TA. 2007. Crescimento, características estruturais e teor de óleo essencial de Mentha suaveolens, cultivada sob telas coloridas. Lavras: UFLA. 67 p (Dissertação mestrado).

BEHN H; ALBERT A; MARX F; NOGA G; ULBRIC A. 2010. Ultraviolet-B and photosynthetically active radiation interactively affect yield and pattern of monoterpenes in leaves of peppermint (Mentha x piperita). Journal of Agricultural and Food Chemistry 58: 7361-7367.

BENINCASA MMP. 2003. Análise de crescimento de plantas. Jaboticabal: FUNEP. 41p.

BURBOTT AJ; LOOMIS WD. 1967. Effects of light and temperature on the monoterpenes of peppermint. Plant Physiology 42: 20-28.

CASTRO EM; GAVILANES ML; ALVARENGA AA; CASTRO DM; GAVILANES TOT. 1998. Aspectos da anatomia foliar de mudas de Guarea guidonea, sob diferentes níveis de sombreamento. Daphne 8: 31-35.

CHAGAS JH; RIBEIRO AS; PINTO JEBP; BERTOLUCCI SKV; BOTREL PP; COSTA AG. 2010a. Análises foliares em plantas de Mentha arvensis cultivada sob diferentes malhas e níveis de sombreamento. Horticultura Brasileira 28: 3464-3471.

CHAGAS JH; RIBEIRO AS; PINTO JEBP; BERTOLUCCI SKV; SANTOS FM; BOTREL PP. 2010b. Acúmulo de biomassa seca em plantas de Mentha arvensis cultivada sob diferentes malhas e níveis de sombreamento. Horticultura Brasileira 28: 3457-3463.

CHANG X; ALDERSON PG; WRIGHT CJ. 2008. Solar irradiance level alters the growth of basil (Ocimun basilicum) and its content of volatile oils. Enviromental and Experimental Botany 63: 216-223.

CORRÊA RM. 2008. Adubação orgânica, intensidade e qualidade de luz no crescimento de plantas, características anatômicas e composição química do óleo essencial de orégano (Origanum vulgare). Lavras: UFLA. 131p. (Tese mestrado).

COSTA AG; CHAGAS JH; PINTO JEBP BERTOLUCCI SKV. 2012. Crescimento vegetativo e produção de óleo essencial de hortelã-pimenta cultivada sob malhas. Pesquisa Agropecuária Brasileira 47: $534-$ 540.

DOLZHENKO Y; BERTEA CM; OCCHIPINTI A; BOSSI S; MAFFEI ME. 2010. UV-B modulates the interplay between terpenois and flavonoids in peppermint (Mentha x piperita). Journal of Photochemistry and Photobiology 100: 67-75.

EMPRESA BRASILEIRA DE PESQUISA AGROPECUÁRIA. 1997. Centro Nacional de Pesquisa de Solos (Rio de Janeiro, RJ). Manual de métodos de análise de solo. 2 ed. rev. e atual. Rio de Janeiro: EMBRAPA CNPS. 212 p.

FERREIRA DF. 2007. SISVAR 5.0: sistema de análise estatística. Lavras: UFLA/DEX, Software.

LORENZO D; PAZ D; DELLACASSA E;
DAVIES P; VILA R; CANIGUERAL S. 2002. Essential oil of Mentha pulegium and Mentha rotundifolia from Uruguai. Brazilian Archives of Biology Technology 45: 519-524.

MAFFEI M; CANOVA D; BERTEA CM; SCANNERINI S. 1999. UV-A effects on photomorphogenesis and essential-oil composition in Mentha piperita. Journal of Photochemistry and Photobiology 52:105-110.

MAHMOUD SS; CROTEAU RB. 2003. Menthofuran regulates essential oil biosynthesis in peppermint by controlling a downstream monoterpene reductase. Proceedings of the National Academy of Sciencesof the United States of America 100: 14481-14486.

MARTINS JR; ALVARENGAAA; CASTRO EM; PINTO JEBP; SILVA APO. 2008. Avaliação do crecimento e teor do óleo essencial em plantas de Ocimum gratissimum cultivadas sob malhas coloridas. Revista Brasileira de Plantas Medicinais 10: 102-107.

NOBEL PS. 1991. Physicochemical and environmental plant physiology. San Diego: Academic Press. 635 p.

PEGORARO RL; FALKENBERG MB; VOLTOLINI CH; SANTOS M; PAULILO MTS. 2010. Produção de óleos essenciais em plantas de Mentha x piperita var. piperita (Lamiaceae) submetidas a diferentes níveis de luz e nutrição do substrato. Revista Brasileira de Botânica 33: 631-637.

POLYSACK INDÚSTRIAS Ltda. 2008. Disponível em: http://www.polysack.com/ files/fc42f05f6\%20bab9497f30102\%20 a306073755.pdf Acessado em: 6 de novembro de 2008.

SACCO T. 1987. Comportamento della Mentha piperita al fotoperiodismo de1 22 lat. Sud. (Brasile). Essenze Derivati Agrumari 57: 535-539.

SCALON SPQ; SCALON FILHO H; RIGONI MR; VERALDO F. 2001. Germinação e crescimento de mudas de pitangueira (Eugenia uniflora) sob condições de sombreamento. Revista Brasileira de Fruticultura 23: 1-5.

VISCHI FILHO OJ. 2002. Avaliação de casas de vegetação para fins quarentenários de flores, com diferentes graus de automação. Campinas: UNICAMP. 151p. (Dissertação mestrado). 